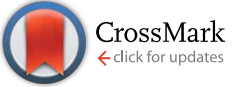

Cite this: RSC Adv., 2016, 6, 113432

Received 15th September 2016 Accepted 31st October 2016

DOI: 10.1039/c6ra23059h

www.rsc.org/advances

\section{Surface modification and porosimetry of vertically aligned hexagonal mesoporous silica films $\uparrow$}

\author{
Calum Robertson, a Andrew W. Lodge, ${ }^{a}$ Peter Basa, ${ }^{b}$ Marina Carravetta, ${ }^{a}$ \\ Andrew L. Hector, ${ }^{* a}$ Reza J. Kashtiban, ${ }^{c}$ Jeremy Sloan, ${ }^{C}$ David C. Smith, ${ }^{d}$ \\ Joseph Spencer ${ }^{d}$ and Alain Walcarius ${ }^{e}$
}

Mesoporous silica films with vertically aligned hexagonal pores have been produced via the electrochemically assisted surfactant assembly (EASA) method using cetyltrimethylammonium bromide (CTAB) surfactant. Mesoporous silica powder has also been synthesised using the same surfactant. The pore walls of the silica powder and films have been grafted with organosilane reagents. The size of the pore, degree of grafting and effect on the properties of the pore have been investigated using porosimetry, contact angle, NMR and CHN analysis. The degree of grafting was found to be dependent upon the size of the grafting agent, with the smallest steric bulk grafting most effectively. It was found that the grafting of the pores with $\mathrm{Me}_{3} \mathrm{SiCl}$ greatly increased the hydrophobicity of the pore and reduced water penetration. Grafting with larger groups caused the film surface to be hydrophobic but had little effect on the penetration of water into the pores.

\section{Introduction}

Mesoporous silica powders can readily be produced with various pore sizes and with $1 \mathrm{D}$ or 3D pore structures, and hence provide a chemically stable platform for applications in catalysis, adsorption and sensors. ${ }^{1-4}$ An important aspect of their utility is the ability to change the surface chemistry of the pores by addition of reagents containing direct $\mathrm{Si}-\mathrm{R}$ bonds $(\mathrm{R}=$ substituent) to the silica synthesis procedure..$^{5-8}$ An early example of modification of the pore walls after synthesis of the silica ("grafting") used $\mathrm{Me}_{3} \mathrm{SiCl}$ to replace the hydroxyl groups on the pore walls with trimethylsilyl groups, causing a decrease in the diameter of the pore. ${ }^{9}$ This also replaces the relatively hydrophilic silica surface with a hydrophobic one terminated by $\mathrm{C}-\mathrm{H}$ bonds. Using substituents that contain functional groups it has also been possible to produce selective catalysts, ${ }^{\mathbf{1 0}}$ sensors for humidity, $\mathrm{pH}$ and metal ions, ${ }^{\mathbf{1 1}}$ proton conductors, ${ }^{\mathbf{1 2}}$

${ }^{a}$ Chemistry, University of Southampton, Highfield, Southampton, SO17 1BJ, UK. E-mail: A.L.Hector@soton.ac.uk

${ }^{b}$ Semilab Semiconductor Physics Laboratory Co. Ltd., Prielle Kornélia str. 2, H-1117 Budapest, Hungary

'Department of Physics, University of Warwick, Coventry CV4 7AL, UK

${ }^{d}$ Physics and Astronomy, University of Southampton, Highfield, Southampton, SO17 $1 B J, U K$

${ }^{e}$ Laboratoire de Chimie Physique et Microbiologie pour l'Environnement, UMR 7564 CNRS - Université de Lorraine, 405, rue de Vandoeuvre, 54600 Villers-les-Nancy, France

$\dagger$ Electronic supplementary information (ESI) available. See DOI: 10.1039/c6ra23059h

\$ Raw data are also available from DOI: 10.5258/SOTON/402889. electrochemical sensors ${ }^{\mathbf{1 3}}$ and biosensors, ${ }^{\mathbf{1 4}}$ and absorbents to remove contaminants from water ${ }^{15,16}$ or air. ${ }^{17}$

Mesoporous silica films are commonly synthesised via the evaporation induced self-assembly (EISA) method, ${ }^{18-21}$ using solutions of tetraethyl orthosilicate (TEOS) and a surfactant in a water/ethanol sol. In the EISA method, the sol is dip-coated onto the substrate and as the ethanol evaporates the surfactant concentration increases, causing the formation of micelles, around which the silicates condense. The pore diameter of the resultant silica is determined by the size of the micelles, and their orientations by interactions between micelle surfaces and neighbouring micelles or the substrate. Vertical alignment is very difficult to achieve, but with cationic surfactants such as cetyltrimethylammonium bromide (CTAB) hexagonally packed arrays of vertically aligned mesopores can be obtained by the electrochemically assisted surfactant assembly (EASA) method $^{2-27}$ (alternatives are Stöber solution growth or oilinduced co-assembly methods ${ }^{28,29}$ ). EASA uses an electronically conductive substrate immersed in the sol. The application of a negative potential causes the self-assembly of the cationic surfactant and the passing of the current results in the formation of hydroxide ions close to the substrate surface. ${ }^{26,30,31}$ This $\mathrm{pH}$ change catalyses the condensation of the silicate and porous silica structures form around the micelles. ${ }^{32}$ For both methods, once the porous structure has been formed the surfactant is removed by pyrolysis or Soxhlet extraction leaving empty pores. ${ }^{27}$ EASA films have been suggested for applications as electrochemical sensors, biosensors and adsorbents. ${ }^{\mathbf{1 3 , 1 4 , 1 6}}$

Our interest in surface modification of EASA films is related to the possibility of using them as templates for supercritical 
fluid electrodeposition to grow arrays of very small diameter nanowires. ${ }^{27,33-35}$ Supercritical fluids can effectively be used to penetrate small pores due to their low viscosities and zero surface tension. Previously we showed that EASA films on titanium nitride, a substrate chosen for its compatibility with a wide range of electrodeposition conditions ${ }^{34,36}$ and with contacting of many functional nanowire materials, results in pore diameters of $\sim 1.6 \mathrm{~nm} .{ }^{27}$ Grafting of these pores may provide changes in pore size, access of dissolved species to the electrodeposition growth surface or modifications to the properties of nanowires grown in the templates. Previous work on the modification of EASA films has involved co-condensation of TEOS with an organosilane ${ }^{26,30,31}$ or coupling this approach to "Click" chemistry to modify the silica surface. ${ }^{37,38}$ Post synthesis functionalisation has also been demonstrated with hydroquinone derivatives. ${ }^{39}$ In this paper, the effects of several grafting agents on the properties of EASA silica films is examined, with studies of the same chemistries on mesoporous silica powders used to provide further insight.

\section{Experimental}

A one-pot synthesis of mesoporous silica powder was derived from the work of Nooney et al. ${ }^{4}$ A solution of $40.7 \mathrm{~cm}^{3}$ aqueous ammonia ( $37 \% \mathrm{w} / \mathrm{w}$, Fisher) in $442 \mathrm{~cm}^{3} \mathrm{H}_{2} \mathrm{O}$ was heated to $50{ }^{\circ} \mathrm{C}$ whilst stirring in a round-bottomed flask. CTAB $(1.10 \mathrm{~g}$, SigmaAldrich) was dissolved in the solution, TEOS $\left(5.6 \mathrm{~cm}^{3}, 98 \%\right.$, Sigma-Aldrich) was added, and the solution was stirred whilst being allowed to cool to room temperature over two hours. The precipitate was filtered under suction and the remaining powder was dried at $60{ }^{\circ} \mathrm{C}$ for 16 hours. The surfactant was removed from the silica by washing the powder in a Soxhlet extraction thimble with $0.1 \mathrm{M} \mathrm{HCl} / \mathrm{EtOH}$ for 6 hours.

MOCVD titanium nitride coated silicon wafers were purchased from Si-Mat. The wafers were supplied with a PVD silica capping layer and a $0.8 \mathrm{~cm}$ diameter circular region was etched into the silica using aqueous HF to provide a deposition surface with the remainder of the electrode insulated by the silica film. Mesoporous silica films were produced on TiN films using the electrochemically assisted surfactant assembly (EASA) method. A $10 \mathrm{mM}$ solution of $\mathrm{NaNO}_{3}$ in $\mathrm{H}_{2} \mathrm{O}\left(20 \mathrm{~cm}^{3}\right)$ was added to $20 \mathrm{~cm}^{3}$ of ethanol. $0.2 \mathrm{M} \mathrm{HCl} / \mathrm{H}_{2} \mathrm{O}$ was added dropwise to the solution until the $\mathrm{pH}$ was approximately 3 . TEOS $\left(0.905 \mathrm{~cm}^{3}\right)$ was added to the solution and allowed to stir for 90 minutes. CTAB $\left(0.47 \mathrm{~g}, 1.29 \times 10^{-3} \mathrm{~mol}\right)$ was added to the solution and allowed to stir for 30 minutes until fully dissolved. A cone shaped PTFE electrochemical cell was equipped with a steel counter electrode, a $3 \mathrm{~mm}$ diameter silver rod pseudo-reference electrode, and the TiN coated $\mathrm{Si}$ wafer working electrode. A potential of $-1.3 \mathrm{~V} v s$. $\mathrm{Ag} / \mathrm{Ag}^{+}$was applied for $20 \mathrm{~s}$. The films were immediately removed from the solution and washed with water before being dried in air overnight at $130{ }^{\circ} \mathrm{C}$. The CTAB surfactant was removed from the mesopores by Soxhlet extraction over $5 \mathrm{~h}$ using $0.2 \mathrm{M} \mathrm{HCl} / \mathrm{EtOH}$.

For grafting, the mesoporous silica powder $(0.5 \mathrm{~g})$, or an EASA silica film on a silicon/TiN wafer, was first dried at $110{ }^{\circ} \mathrm{C}$ under vacuum for $6 \mathrm{~h}$ then handled under nitrogen to avoid introducing excess moisture that could react with the grafting agents to block pores. The grafting agents that were used were trimethylchlorosilane $\left(\mathrm{Me}_{3} \mathrm{SiCl}\right)$, phenyldimethylchlorosilane $\left(\mathrm{PhMe}_{2} \mathrm{SiCl}\right)$, tert-butyldimethylchlorosilane $\left({ }^{t} \mathrm{BuMe}_{2} \mathrm{SiCl}\right)$ and hexamethyldisilazane $\left(\mathrm{HN}\left(\mathrm{SiMe}_{3}\right)_{2}\right)$, and all were obtained from Sigma-Aldrich and used as supplied. The powder or film was placed in $10 \mathrm{~cm}^{3}$ of dry THF and $3.9 \mathrm{mmol}$ of the grafting agent was added, followed by refluxing for $24 \mathrm{~h}$. The solution was removed and the sample was washed with THF $\left(5 \times 20 \mathrm{~cm}^{3}\right)$ and diethyl ether $\left(5 \times 20 \mathrm{~cm}^{3}\right)$ followed by Soxhlet extraction for 6 hours with diethyl ether followed by drying under vacuum at $110{ }^{\circ} \mathrm{C}$.

Small angle X-ray scattering (SAXS) was performed on a Rigaku Smartlab Thin Film instrument using a $9 \mathrm{~kW} \mathrm{Cu}-\mathrm{K}_{\alpha}$ source and a DTex250 1D detector. Powder samples were mounted inside $0.7 \mathrm{~mm}$ diameter borosilicate glass capillaries and measured from $1^{\circ}$ to $10^{\circ} 2 \theta$. For thin films, grazing incidence (GI)SAXS scans were collected from 1 to $10^{\circ} 2 \theta$ using a $0.25^{\circ}$ incidence angle and either in-plane or out-ofplane geometry. Transmission electron microscopy (TEM) was conducted in a JEOL $2100 \mathrm{LaB}_{6}$ instrument operating at $200 \mathrm{kV}$.

${ }^{29}$ Si MAS-NMR measurements were performed on a wide bore 14.1 T Bruker Avance II spectrometer on a $4 \mathrm{~mm}$ probe with a spinning frequency of $8 \mathrm{kHz}$. The spectra were acquired using cross polarisation (CP) and 1024 scans per sample, and referenced using silicone rubber at $-22.3 \mathrm{ppm}$ as indirect reference. Additional ${ }^{29} \mathrm{Si}$ data were acquired using direct excitation with a pulse delay of $600 \mathrm{~s}$, and in the $\mathrm{Me}_{3} \mathrm{Si}$-grafted sample pulse delays of $900 \mathrm{~s}$ were also used to check that sites were fully relaxed and hence that the relative intensities of features were fairly quantitative. ${ }^{13} \mathrm{C}$ MAS NMR data were acquired using $1024 \mathrm{CP}$ scans. The axis was calibrated using adamantane at $-38.5 \mathrm{ppm}$ as indirect reference.

Nitrogen adsorption-desorption measurements were performed on powder samples using a Micromeritic 3Flex Surface Characterisation Analyser. The powder samples were dehydrated prior to measurement by heating to $110^{\circ} \mathrm{C}$ under vacuum overnight to remove any water. The samples were analysed at $77 \mathrm{~K}$. Surface area was determined by the Brunauer-Emmett-Teller (BET) method. ${ }^{40}$ The pore size distribution was modelled using a non-local density-functional theory (NLDFT) approach ${ }^{41-44}$ implemented within the Micromeritics SAIEUS software package (Solution of Adsorption Integral Equation Using Splines). Porosimetry on thin film samples used a PS-2000 ellipsometric porosimeter, with samples exposed to water, toluene, isopropanol or methanol vapour with ellipsometry incidence angles of $60^{\circ}$. The BET method was again used to model surface area, with a modified Kelvin equation or the Dubinin-Rasushkevich model used to calculate the pore size distribution. ${ }^{45,46}$ The substrate contribution was modelled on a plain wafer of MOCVD TiN-coated silicon.

Water contact angle measurements were made using digital photographs of a $1 \mu \mathrm{L}$ water droplet on the surface. Combustion analysis (for $\mathrm{C}, \mathrm{H}$ and $\mathrm{N}$ ) was outsourced to Medac Ltd. and used a Thermo FlashEA ${ }^{\circledR} 1112$ elemental analyser. 


\section{Results and discussion}

Mesoporous silica powders and EASA films were produced with hexagonal pore structure (space group $P 6 \mathrm{~mm}$ ) and a similar lattice spacing. This allowed the same grafting procedures to be applied to both, providing access to a broad range of analytical techniques that can only be applied to powder samples and allowing comparisons between the two systems.

\section{Mesoporous silica powders}

Hexagonal mesoporous silica was produced from TEOS and CTAB using an adaptation of a published route. ${ }^{4}$ SAXS measurements on these samples (Fig. 1) showed the $10\left(2.28^{\circ}\right)$, $11\left(3.92^{\circ}\right)$ and $20\left(4.52^{\circ}\right)$ reflections of hexagonal mesoporous silica with a pore spacing equal to the lattice parameter of $a=$ $4.50(1) \mathrm{nm}$. The porosity of the unmodified mesoporous silica powders was analysed using nitrogen sorption (Fig. 2). The isotherms adopted the IUPAC type IV profile that is typical for these MCM-41 type materials, with similar amounts of monolayer adsorption on the silica surfaces (low pressure) and pore filling $\left(P / P_{0}=0.2-0.4\right)$ as reported previously. ${ }^{4}$ The BET analysis measured the surface area as being $1093 \mathrm{~m}^{2} \mathrm{~g}^{-1}$ and the pore size distribution calculated using NLDFT was centred on $2.95 \mathrm{~nm}$ (Fig. 2). Classically the analysis of pore sizes in gas sorption data used the Barrett-Joyner-Halenda (BJH) method, but it has been shown this approach underestimates pore sizes in small mesopores and in the microporous regimes. ${ }^{47}$ The NLDFT approach used herein was developed by Seaton et al., ${ }^{\mathbf{4 4}}$ received ISO standardisation in $2007,{ }^{43}$ and has been found to be reliable when used on MCM-41 silicas. $^{48-50}$

\section{EASA mesoporous silica films with vertically aligned pores}

We previously reported the EASA deposition of mesoporous silica films on PVD TiN films. ${ }^{27}$ Herein the substrate type was changed to a commercial MOCVD TiN with higher electronic conductivity, and this affected the deposition conditions under which the EASA films could be deposited to a surprising degree. Depositions under different conditions chosen for

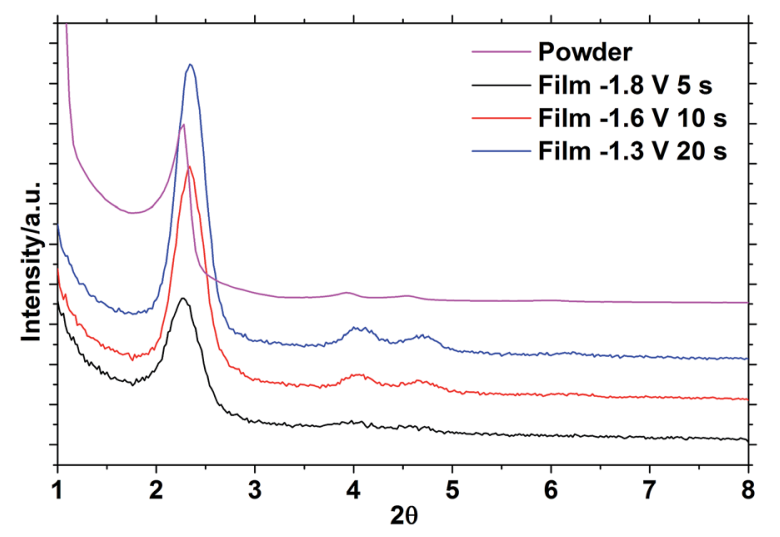

Fig. 1 The SAXS pattern of mesoporous silica powder and the in-plane GISAXS patterns of mesoporous silica films deposited at conditions as labelled.
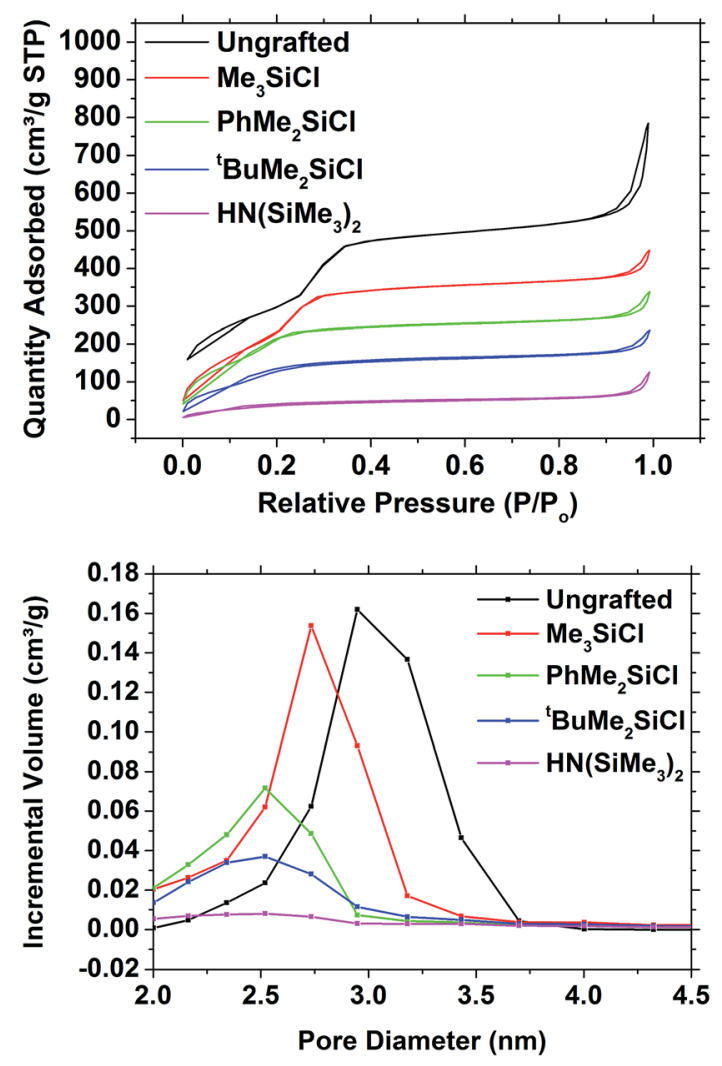

Fig. 2 Nitrogen adsorption/desorption isotherms (top) and NLDFT pore size distribution (bottom) of mesoporous silica powder and of grafted mesoporous silica powders.

roughly the same amount of charge to have been passed resulted in the GISAXS patterns shown in Fig. 1 and analysed in Table 1. The 10, 11, 20 and 21 reflections associated with a hexagonal $(P 6 \mathrm{~mm})$ pore structure were clearly visible in the in-plane GISAXS, whereas only very broad, weak features close to the position of the 10 reflection were observed out-of-plane. This confirms that the pores are vertically aligned with negligible horizontal alignment. The pore spacing of $4.36(2) \mathrm{nm}$ observed in these films is also similar to that observed in our previous work, ${ }^{27}$ where the vertical pore structure was also extensively characterised by electron microscopy, and in the powdered silica $(4.50(3) \mathrm{nm})$. The most intense GISAXS pattern was obtained after a deposition held at $-1.3 \mathrm{~V}$ for $20 \mathrm{~s}$, so these conditions were chosen to produce the samples used in subsequent studies. An exemplar TEM image of one of these films, confirming the pore orientation, is shown in Fig. S1.†

Table 1 Peaks in the in-plane GISAXS patterns (Fig. 1) of EASA films deposited on TiN under various conditions, and calculated lattice parameters (potentials quoted vs. $\mathrm{Ag} / \mathrm{Ag}^{+}$)

\begin{tabular}{llllll}
\hline Deposition conditions & $2 \theta(10) /{ }^{\circ}$ & $2 \theta(11) /{ }^{\circ}$ & $2 \theta(20) /{ }^{\circ}$ & $2 \theta(21) /{ }^{\circ}$ & $a / \mathrm{nm}$ \\
\hline$-1.8 \mathrm{~V}, 5 \mathrm{~s}$ & 2.28 & 3.98 & 4.62 & - & $4.44(2)$ \\
$-1.6 \mathrm{~V}, 10 \mathrm{~s}$ & 2.34 & 4.04 & 4.68 & 6.20 & $4.364(7)$ \\
$-1.3 \mathrm{~V}, 20 \mathrm{~s}$ & 2.34 & 4.06 & 4.70 & 6.18 & $4.351(7)$
\end{tabular}


Ellipsometric porosimetry can be used to provide information on the pore structure of thin films, ${ }^{45,51}$ performing the same role as nitrogen adsorption/desorption measurements with powders. These measurements are performed with a sample placed in a sealed chamber and a laser is reflected off the surface of the thin-film sample. The initial measurements are taken under vacuum conditions and the refractive index of the film is determined. The humidity in the chamber is then increased and the refractive index is continually measured. As the film is exposed to vapour of a probe molecule such as water or toluene, condensation occurs within the porous structure of the film and causes a change in the refractive index of the film. By monitoring the change in the refractive index of the sample as the partial pressure is increased, the absorption/desorption isotherm can be determined.

The pore structure of the EASA silica films was analysed by ellipsometric porosimetry using water, toluene, isopropyl alcohol and methanol probe molecules. All four probe molecules produced type IV isotherms (Fig. 3), with the partial pressures at which capillary condensation occurs varying roughly with the polarity of the probe molecule. Toluene wets the pores and condenses at low pressure, but desorbs cleanly over the same pressure range, returning to its original refractive index at low pressure. The alcohol and water isotherms exhibit hysteresis due to hydrogen bonding to the pore wall hydroxyl groups. Unsurprisingly this effect is strongest with water, where incomplete removal of the water by the vacuum is also apparent from a higher final refractive index at the end of the desorption process than at the start of the experiment. A large hysteresis was previously observed in ellipsometric porosimetry of EASA films on ITO using water vapour. ${ }^{52}$ Porosities of the EASA films calculated from the adsorption branches of these isotherms were $38.2 \%$ using toluene, $40.1 \%$ using water, $42.6 \%$ using isopropyl alcohol and $42.5 \%$ using methanol. These are in good mutual agreement, although clearly some variability between films is a clear possibility. Powders produced with a CTAB surfactant have pore sizes in the range 3.0-5.7 $\AA$, depending on a range of factors during the synthesis., ${ }^{\mathbf{4 5 3}-55}$ The electrochemical EASA growth method results in smaller pores, with $1.6 \mathrm{~nm}$ pores found in EASA silica films on TiN. ${ }^{27}$ A $4.46 \mathrm{~nm}$ pore spacing combined with this pore size would result in a porosity of $31 \%$.

Analysis of surface area and pore size was considered to be most effective with the least polar solvents, where both the adsorption and desorption branches could be used. It has been argued that porous confinement can distort results using a polar solvent due to the greater degree of polarisation present in the surface/adsorbate interaction. ${ }^{56}$ The BET surface area of the film was determined to be $1093 \mathrm{~m}^{2} \mathrm{~g}^{-1}$ using the toluene isotherm. Application of a modified Kelvin equation ${ }^{45}$ to the toluene isotherm led to a pore diameter of $2.72 \mathrm{~nm}$ using the adsorption branch or $2.74 \mathrm{~nm}$ using the desorption. This is higher than previously observed in EASA silica ${ }^{27}$ but it has been previously acknowledged that the Kelvin equation ignores intermolecular interactions that become increasingly important at small pore size, and hence is likely to overestimate the pressure required to wet pores in the micropore regime $(<2 \mathrm{~nm}$ (ref. 46)). The Dubinin-Rasushkevich (DR) model assumes that liquid in the pore channels is homogeneously filled and has liquid-like properties, and hence becomes more accurate as the intermolecular interactions become dominant in the micropore region. ${ }^{57}$ Note that the samples studied herein were expected to have a pore size at the upper end of this regime. Using the DR model with the toluene isotherm (Fig. 4) the pore size was determined to be $1.44 \mathrm{~nm}$ (adsorption branch) or $1.48 \mathrm{~nm}$ (desorption branch). Using the isopropyl alcohol isotherms the values were slightly smaller, at $1.14 \mathrm{~nm}$ (adsorption) or $1.20 \mathrm{~nm}$ (desorption), reflecting the larger wall interaction. Hence the toluene data fitted with the DR model were found to provide the most plausible pore size results.

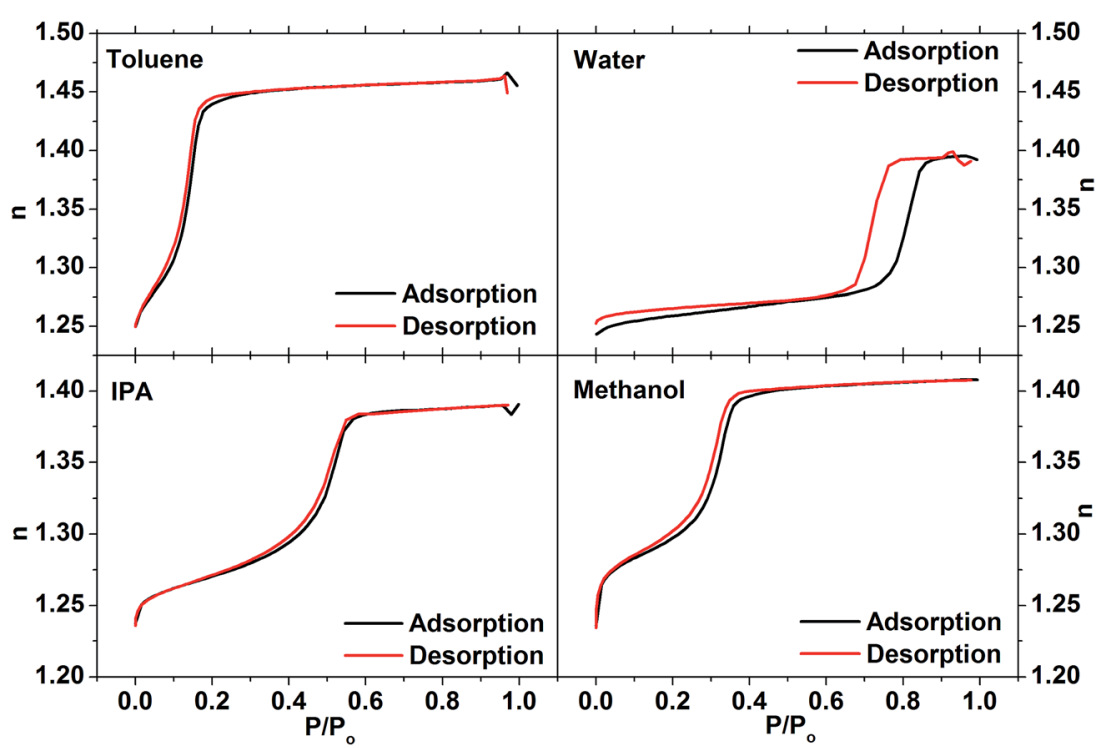

Fig. 3 Plots of refractive index $(n)$ of an EASA mesoporous silica film vs. solvent vapour partial pressure during adsorption and desorption of toluene, water, isopropyl alcohol or methanol. 


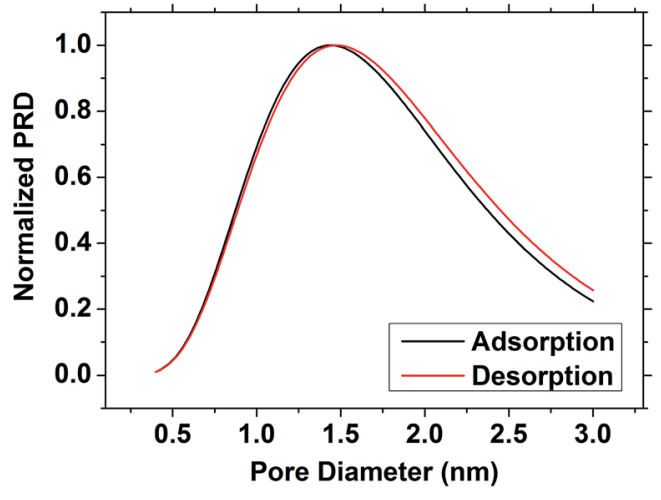

Fig. 4 The pore size distribution of the EASA silica film as determined using the toluene isotherm and the Dubinin-Radushkevich model.

\section{Surface modification of mesoporous silica powders}

Surface treatments of mesoporous silica utilized trialkyl (/aryl) chlorosilanes or hexamethyldisilazane. The dried powder silica samples were refluxed with these compounds in THF. They were then subjected to a thorough washing regime to remove as much of the unreacted grafting agent as possible, including a Soxhlet extraction. The SAXS patterns were essentially indistinguishable from those of the ungrafted silica powder (Fig. S2 $\dagger$ ), so the mesoporous structure underwent no significant changes in the pore ordering. The in-plane GISAXS measurements gave lattice parameters of 4.493(12) $\mathrm{nm}$ for ungrafted powder, $4.441(8) \mathrm{nm}$ for $\mathrm{Me}_{3} \mathrm{SiCl}$ grafted powder, $4.312(8) \mathrm{nm}$ for $\mathrm{PhMe}_{2} \mathrm{SiCl}$ grafted powder, 4.36(2) nm for ${ }^{t} \mathrm{BuMe}_{2} \mathrm{SiCl}$ grafted powder and 4.231(14) $\mathrm{nm}$ for $\mathrm{HN}\left(\mathrm{SiMe}_{3}\right)_{2}$ grafted powders.

The nitrogen adsorption-desorption isotherms (Fig. 2) showed a gradual decrease in the size of the capillary condensation feature as the size of the grafting group increased, and at the same time a reduction both in the pore diameter and the pore volume. The surface area of the samples decreased from the $1093 \mathrm{~m}^{2} \mathrm{~g}^{-1}$ in the ungrafted silica to $904 \mathrm{~m}^{2} \mathrm{~g}^{-1}$ with $\mathrm{Me}_{3} \mathrm{SiCl}, 729 \mathrm{~m}^{2} \mathrm{~g}^{-1}$ with $\mathrm{PhMe}_{2} \mathrm{SiCl}, 538 \mathrm{~m}^{2} \mathrm{~g}^{-1}$ with ${ }^{t} \mathrm{BuMe}_{2}-$ $\mathrm{SiCl}$ and $161 \mathrm{~m}^{2} \mathrm{~g}^{-1}$ with $\mathrm{HN}\left(\mathrm{SiMe}_{3}\right)_{2}$. The NLDFT pore diameter decreased from $2.95 \mathrm{~nm}$ to $2.73,2.52,2.52$ and $2.50 \mathrm{~nm}$ in the same sequence, although a significant loss of pore volume was also observed with the larger agents (Fig. 2), suggesting some pore blocking was also occurring.

The ${ }^{29} \mathrm{Si}$ MAS-NMR spectrum of the ungrafted silica powder (Fig. 5) contained two broad signals at -98.7 and $-107.5 \mathrm{ppm}$, corresponding to the Q3 $\left(\mathrm{Si}(\mathrm{OH})(\mathrm{OSi})_{3}\right)$ and $\mathrm{Q} 4\left(\mathrm{Si}(\mathrm{OSi})_{4}\right)$ environments within the silica. ${ }^{58,59}$ These persisted after grafting (with peak shifts of no more than $1.5 \mathrm{ppm}$ between samples), but were then also accompanied by signals at in the range of 3 to $16 \mathrm{ppm}$ (14.1, 3.6, 16.7 and 12.8 respectively for $\mathrm{Me}_{3} \mathrm{SiCl}$, $\mathrm{PhMe}_{2} \mathrm{SiCl},{ }^{t} \mathrm{BuMe}_{2} \mathrm{SiCl}$ and $\left.\mathrm{HN}\left(\mathrm{SiMe}_{3}\right)_{2}\right)$ corresponding to the grafted silane molecules. ${ }^{59}$ The upfield shift for the $\mathrm{Ph}$ substituted silane and downfield shift for the ${ }^{t} \mathrm{Bu}$ substituted silane are in line with trends observed in the literature. ${ }^{60}$ These are significantly different from the ${ }^{29} \mathrm{Si}$ chemical shifts of the chlorosilane reagents, provided in Blinka et al ${ }^{60}$ for $\mathrm{Me}_{3} \mathrm{SiCl}$,
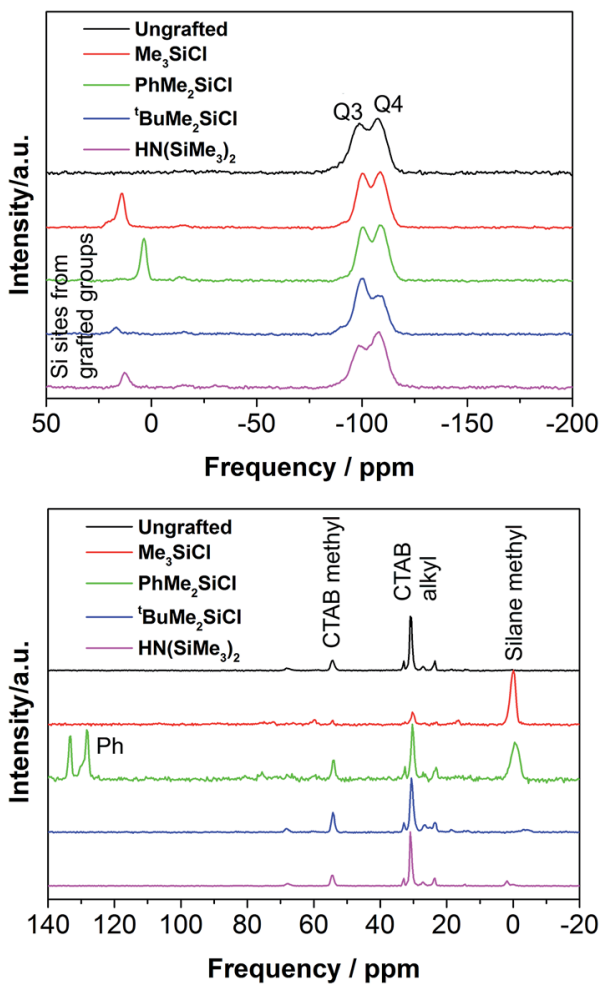

Fig. $5{ }^{29} \mathrm{Si}$ (top) and ${ }^{13} \mathrm{C}$ (bottom) CP MAS-NMR spectra of the mesoporous silica powder samples.

${ }^{t} \mathrm{BuMe}_{2} \mathrm{SiCl}$ and $\mathrm{HN}\left(\mathrm{SiMe}_{3}\right)_{2}$, suggesting that no unreacted materials are present in the systems studied here. The strongest signals are present in the $\mathrm{Me}_{3} \mathrm{SiCl}$ and $\mathrm{PhMe}_{2} \mathrm{SiCl}$ grafted powders, suggesting that these samples contained the largest number of grafted sites. As the $\mathrm{Si}(\mathrm{OH})(\mathrm{OSi})_{3}$ sites are reacted with the grafting agent the Q3 signal in the grafted samples should decrease relative to Q4 and to the Q3 signal in the ungrafted membrane. ${ }^{61}$ The CP spectra (Fig. 5) are not quantitative so direct acquisition spectra were also collected (Fig. S3†) and these confirm the expected decrease in the Q3 signal.

The ${ }^{13} \mathrm{C}$ spectra (Fig. 5) show the presence of some surfactant impurities (signals near $54 \mathrm{ppm}$ for $\mathrm{NMe}_{3}$ group and signals between 20-35 ppm for the long aliphatic chain of CTAB). This indicates that there is still CTAB present in several of the films, but also that the $\mathrm{Me}_{3} \mathrm{SiCl}$ grafting removed almost all of the remaining CTAB from the film. Both ${ }^{29} \mathrm{Si}$ and ${ }^{13} \mathrm{C}$ NMR data suggest that there is no tetraethoxysilane left as unreacted material in the sample. The ${ }^{13} \mathrm{C}$ signal for $\mathrm{Si}-\mathrm{Me}$ groups is assigned to the broad signal near $0 \mathrm{ppm}^{60}$ and it is a clear signature for the successful grafting on the surface. Interestingly, the ${ }^{13} \mathrm{C}$ data correlate well with the ${ }^{29} \mathrm{Si}$ data to indicate that in samples with good grafting levels as detected by ${ }^{29} \mathrm{Si}$ $\mathrm{NMR}\left(\mathrm{Me}_{3} \mathrm{SiCl}\right.$ and $\left.\mathrm{PhMe}_{2} \mathrm{SiCl}\right)$, the corresponding signals from the grafted species are clearly visible by ${ }^{13} \mathrm{C}$ NMR. The $\mathrm{PhMe}_{2}-$ $\mathrm{SiCl}$ species give additional signals near 130-140 ppm. For the other samples $\left({ }^{t} \mathrm{BuMe}_{2} \mathrm{SiCl}\right.$ and $\left.\mathrm{HN}\left(\mathrm{SiMe}_{3}\right)_{2}\right)$ where there is a very weak signal from grafted silicon $\mathrm{via}^{29} \mathrm{Si}$ NMR and the ${ }^{13} \mathrm{C}$ signal from $\mathrm{Si}-\mathrm{Me}$ is also very weak. Both ${ }^{13} \mathrm{C}$ and ${ }^{29} \mathrm{Si}$ data suggests that free starting material for the grafting process 
which did not bond were effectively removed, and do not appear in the ${ }^{13} \mathrm{C}$ or ${ }^{29} \mathrm{Si}$ cross-polarisation spectra.

Combustion analysis showed the ungrafted mesoporous silica powder to contain significant carbon (Table 2). The C: N ratio of CTAB is $19: 1$, similar to the analysed value, so the surfactant seen in the NMR data is likely to be the main contributor. The TEOS starting material or the ethanol used in the Soxhlet extraction of the surfactant could also contribute. Mesoporous silica syntheses often involve firing to burn away the surfactant, but oxidation of the TiN surface of the films precludes this approach. The grafted samples (Table 2) show lower carbon contents and a higher $\mathrm{C}: \mathrm{N}$ ratio, suggesting the surfactant is displaced from the pore during grafting. It is possible that the Soxhlet extraction failed to completely remove the surfactant due to $[\mathrm{CTA}]^{+}$cations balancing surface $\mathrm{SiO}^{-}$ groups, but that these react with the grafting agent releasing the surfactant. $\mathrm{Me}_{3} \mathrm{SiCl}$ and $\mathrm{PhMe}_{2} \mathrm{SiCl}$ show the greatest change in the analysis and were most effective at removing the surfactant, as also shown by the ${ }^{13} \mathrm{C}$ NMR data (Fig. 5). The analysis of the sample grafted with $\mathrm{HN}\left(\mathrm{SiMe}_{3}\right)_{2}$ was close to that of the ungrafted film, indicating that this grafting agent was least successful in releasing surfactant from the pores. The carbon content of $\mathrm{Me}_{3} \mathrm{SiCl}$ and $\mathrm{PhMe}_{2} \mathrm{SiCl}$ grafted films is mainly due to the grafting agent, and taking the number of carbon atoms per grafting molecule into account the coverage of $\mathrm{Me}_{3} \mathrm{Si}$ groups $(11.3 / 3=3.8)$ is seen to be higher than that of $\mathrm{PhMe}_{2} \mathrm{Si}$ groups $(15.8 / 8=1.9)$.

Combined, the NMR and analytical data suggest significant retention of CTAB in the Soxhlet extracted powders. Smaller grafting agents can release this from the pores during the grafting process. The CTAB was not blocking pores significantly according to the ungrafted powder porosimetry, but some reorganisation of the CTAB may result in it blocking pores in conjunction with the larger grafting agents. For example, action of these agents on particle surfaces and pore entrances could result in production of $\mathrm{HCl}$ which could migrate into the pores to release $\mathrm{CTA}^{+}$ions as the chloride salt and protonate the silica. The smaller grafting agents may react simultaneously at sites deeper in the pores and at the surfaces simultaneously, releasing the surfactant and avoiding this effect.

\section{Surface modification of mesoporous silica films}

EASA films were grafted with the same silane reagents as the powders and under the same reaction conditions. In-plane

Table 2 Combustion analysis data from mesoporous silica powder and grafted mesoporous silica powders

\begin{tabular}{llllr}
\hline Grafting agents & $\begin{array}{l}\text { Carbon } \\
(\%)\end{array}$ & $\begin{array}{l}\text { Hydrogen } \\
(\%)\end{array}$ & $\begin{array}{l}\text { Nitrogen } \\
(\%)\end{array}$ & $\begin{array}{l}\text { Ratio of } \\
\text { C/N }\end{array}$ \\
\hline Ungrafted & 26.8 & 5.4 & 1.5 & 17.7 \\
$\mathrm{Me}_{3} \mathrm{SiCl}$ & 11.3 & 2.7 & $<0.1$ & 112.7 \\
$\mathrm{PhMe}_{2} \mathrm{SiCl}$ & 15.4 & 2.6 & 0.2 & 66.9 \\
${ }^{t} \mathrm{BuMe}_{2} \mathrm{SiCl}$ & 18.7 & 3.9 & 0.9 & 20.3 \\
$\mathrm{HN}^{\mathrm{S}}\left(\mathrm{SiMe}_{3}\right)_{2}$ & 24.8 & 5.0 & 1.3 & 19.0
\end{tabular}

GISAXS patterns were very similar to those of the ungrafted films both before and after Soxhlet extraction in acidified ethanol (Fig. S4†). The lattice parameters extracted from these patterns were $4.35(8) \mathrm{nm}$ for an ungrafted silica film, 4.345(16) $\mathrm{nm}$ for a $\mathrm{Me}_{3} \mathrm{SiCl}$ grafted film, 4.39(3) $\mathrm{nm}$ for a $\mathrm{PhMe}_{2} \mathrm{SiCl}$ grafted film and 4.396(18) $\mathrm{nm}$ for a ${ }^{t} \mathrm{BuMe}{ }_{2} \mathrm{SiCl}$ grafted film. The lattice parameters after the films were Soxhlet extracted were unchanged at $4.369(19) \mathrm{nm} \quad\left(\mathrm{Me}_{3} \mathrm{Si}\right)$, $4.345(16) \mathrm{nm}\left(\mathrm{PhMe}_{2} \mathrm{SiCl}\right)$ and $4.384(18) \mathrm{nm}\left({ }^{t} \mathrm{BuMe}_{2} \mathrm{SiCl}\right)$.

Water contact angle measurements were used to determine the surface hydrophobicity of the films. The ungrafted film was fairly hydrophilic, with the water droplet spreading significantly (Fig. 6) and making a contact angle of $61^{\circ}$. The grafted films were all significantly more hydrophobic and the response varied largely with the hydrophobicity of the grafting group, from $84^{\circ}$ with $\mathrm{PhMe}_{2} \mathrm{SiCl}$, to $89^{\circ}$ or $90^{\circ}$, respectively, when grafted with $\mathrm{Me}_{3} \mathrm{SiCl}$ or $\mathrm{HN}\left(\mathrm{SiMe}_{3}\right)_{2}$, and $97^{\circ}$ when grafted with ${ }^{t} \mathrm{BuMe}{ }_{2} \mathrm{SiCl}$ (Fig. 6). The TiN substrate had a contact angle of $79^{\circ}$. This shows that all four grafting agents were effective at modifying the top surface of the films, but does not give an indication of their effectiveness at modifying the pore walls. Hence ellipsometric porosimetry was also employed to probe this. The presence of CTAB in the pores of several of the films, as indicated by the ${ }^{13} \mathrm{C}$ NMR and the combustion analysis, is a potential cause of the hydrophobic character of the surface of the membranes. However the degree of hydrophobicity is smallest in the ungrafted membrane, which contains the highest amount of CTAB. The degree of hydrophobicity is also greater in the $\mathrm{Me}_{3} \mathrm{SiCl}$ grafted membrane, where the grafting agent removed all of the CTAB from the pores. This shows that the CTAB has little effect on the surface hydrophobicity of the films.

Since the $\mathrm{HN}\left(\mathrm{SiMe}_{3}\right)_{2}$ had been found ineffective at grafting the surfaces of the larger pores in the powder samples, the ellipsometric porosimetry studies on the thin films concentrated on the three chlorosilane reagents. With toluene the three films all exhibited the same basic shape of isotherm as found in the ungrafted film (Fig. 7). This is unsurprising since toluene wetted the silica films and would also be expected to wet the more hydrophobic grafted films. The porosity (Table 3) was measured with toluene as $30.4 \%$ with $\mathrm{Me}_{3} \mathrm{SiCl}, 29 \%$ with the larger $\mathrm{PhMe}_{2} \mathrm{SiCl}$ and $37.3 \%$ with ${ }^{t} \mathrm{BuMe}_{2} \mathrm{SiCl}$. This final value was closer to the $38.2 \%$ measured on an ungrafted film.

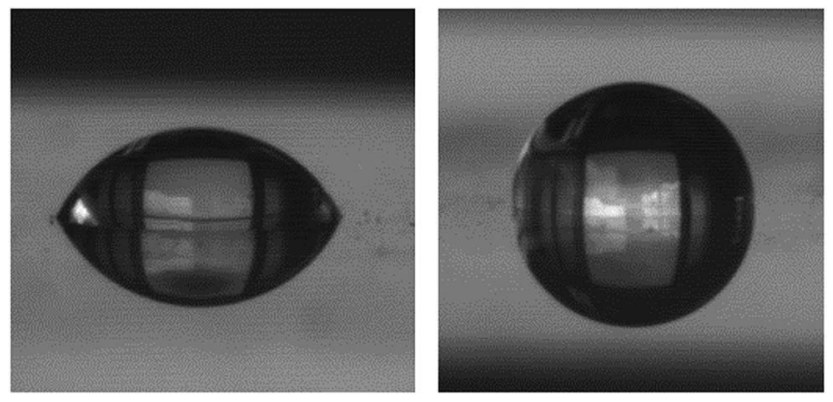

Fig. 6 Images of $1 \mu \mathrm{L}$ water droplets on EASA silica films ungrafted (left) and grafted with $\mathrm{Me}_{3} \mathrm{SiCl}$ (right) for the purpose of contact angle measurement. 


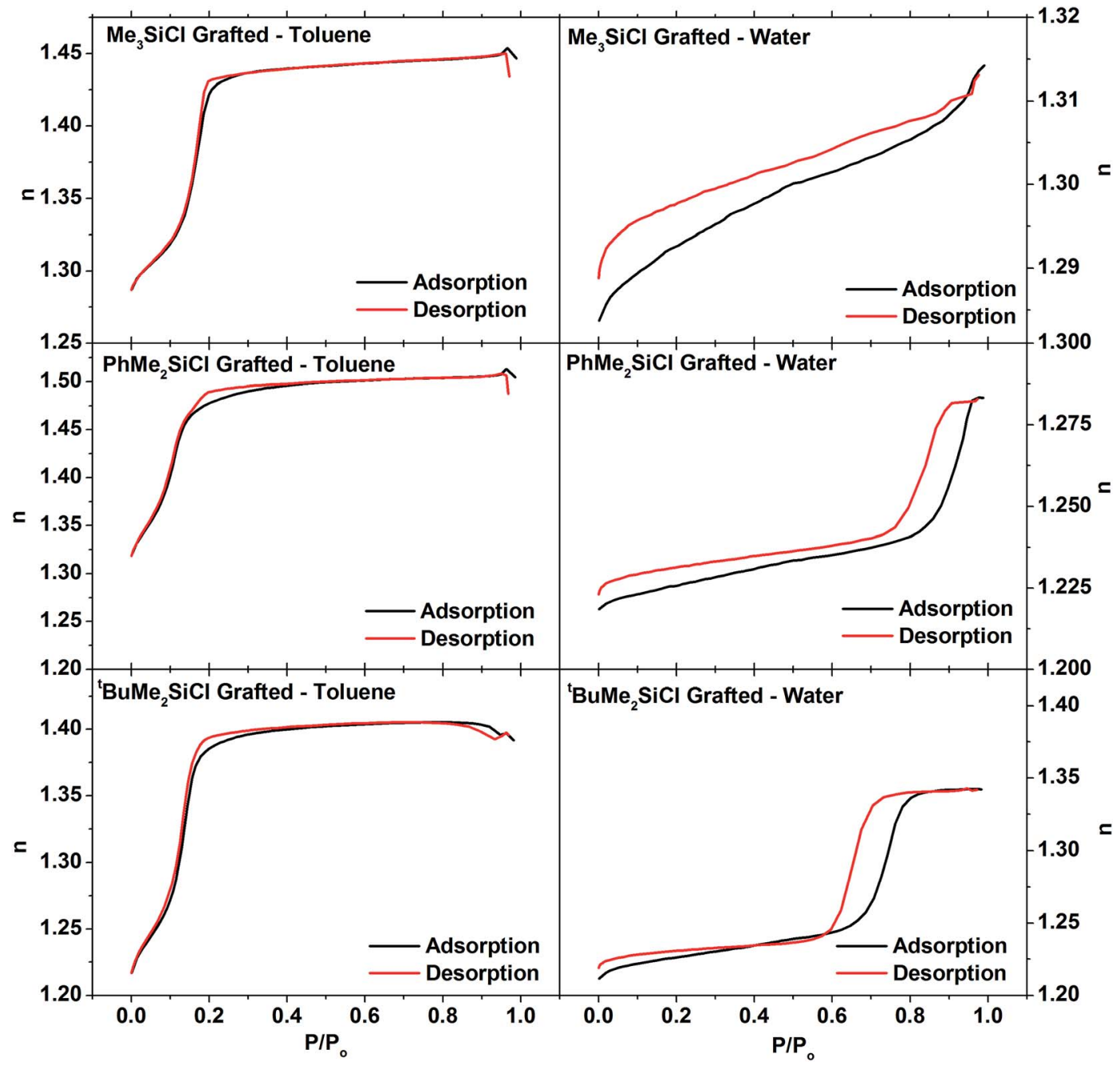

Fig. 7 Ellipsometric porosimetry isotherms measured with toluene (left) or water (right) of EASA silica films grafted with $\mathrm{Me}_{3} \mathrm{SiCl}$ (top), $\mathrm{PhMe}{ }_{2} \mathrm{SiCl}$ (middle) or ${ }^{t} \mathrm{BuMe}{ }_{2} \mathrm{SiCl}$ (bottom).

Interestingly the isotherm with $\mathrm{PhMe}_{2} \mathrm{SiCl}$ exhibited two clear features in the capillary evaporation region of the desorption isotherm and this feature was even more pronounced in the methanol and isopropanol isotherms (Fig. S5-S7 $\dagger$ ), suggesting the presence of two pore diameters. The lack of this feature in the adsorption isotherms suggests that these are not separate pores, but that the top part of the pores is narrowed due to grafting only the most accessible region. The bottom regions of the pores may be less accessible to the larger reagents. Grafting at pore entrances has also been observed in silica powders. ${ }^{62-64}$ Pore diameters measured with toluene (Table 3) were found to increase after grafting, consistent with surfactant removal as seen in the powders.

The isotherm of the $\mathrm{Me}_{3} \mathrm{SiCl}$-grafted film with water shows virtually no capillary condensation and only a very small volume of absorbed vapour at pressures beneath the bulk water vapour pressure. Reconciling these results with the toluene results on the same film requires that a significant fraction, $\approx 75 \%$, of the pores within the treated film are now hydrophobic. The $\mathrm{PhMe}_{2}-$ SiCl grafted film also shows a reduction in pore fraction accessible to water at pressure beneath the bulk water vapour pressure although in this case only $\approx 40 \%$ of the pores are hydrophobic. On the other hand, the ${ }^{t} \mathrm{BuMe}_{2} \mathrm{SiCl}$ treated films show only a very small difference in the porosities measured with water and the other three solvents which suggests very little change in the hydrophilicity of the pores in this film. This is consistent with the poor coverage and surfactant removal observed with this grafting agent in powders. The results also help to explain variable effects on the behaviour of hydrophilic redox probes in EASA films with different surface treatments. ${ }^{37,39}$

Overall it is clear that the grafting process fundamentally preserves the pore structure of the mesoporous film for all of the grafting agents as all of the grafted films show significant porosity when measured with toluene. The smallest grafting agent, $\mathrm{Me}_{3} \mathrm{SiCl}$, shows the clearest evidence of significant pore modification, with the porosity measured using water being only $25 \%$ of the porosity measured using toluene. $\mathrm{PhMe}_{2} \mathrm{SiCl}$, which is slightly larger, also causes a significant change in the hydrophilicity of the pores however to a lesser extent than $\mathrm{Me}_{3} \mathrm{SiCl}$; water porosity is $60 \%$ of the toluene porosity. In the case of ${ }^{t} \mathrm{BuMe}_{2} \mathrm{SiCl}$ 
Table 3 Calculated parameters from ellipsometric porosimetry measurements on mesoporous silica films grafted with various organic groups. Pore diameters were calculated with toluene and IPA where pore wetting was most effective

\begin{tabular}{lllll}
\hline \multirow{2}{*}{ Grafting agent } & Solvent & $\begin{array}{l}\text { Porosity } \\
(\%)\end{array}$ & $\begin{array}{l}\text { Mod. Kelvin eqn } \\
\text { diameter }(\mathrm{nm})\end{array}$ & $\begin{array}{l}\text { DR diameter } \\
(\mathrm{nm})\end{array}$ \\
\hline \multirow{2}{*}{ Ungrafted } & Toluene & 38.2 & $2.72_{\mathrm{abs}} / 2.74_{\mathrm{des}}$ & $1.44_{\mathrm{abs}} / 1.48_{\mathrm{des}}$ \\
& Water & 40.1 & - & - \\
& IPA & 42.6 & $2.74_{\mathrm{abs}} / 2.68_{\mathrm{des}}$ & $1.14_{\mathrm{abs}} / 1.20_{\mathrm{des}}$ \\
& Methanol & 42.5 & - & - \\
$\mathrm{Me}_{3} \mathrm{SiCl}$ & Toluene & 30.4 & $3.14_{\mathrm{abs}} / 3.14_{\mathrm{des}}$ & $1.54_{\mathrm{abs}} / 1.52_{\mathrm{des}}$ \\
& Water & 7.7 & - & - \\
& IPA & 30.7 & $2.86_{\mathrm{abs}} / 2.84_{\mathrm{des}}$ & $1.30_{\mathrm{abs}} / 1.36_{\mathrm{des}}$ \\
& Methanol & 29.26 & - & - \\
$\mathrm{PhMe}_{2} \mathrm{SiCl}$ & Toluene & 29.0 & $2.50_{\mathrm{ads}} / 2.44_{\mathrm{des}}$ & $1.68_{\mathrm{abs}} / 1.76_{\mathrm{des}}$ \\
& Water & 18.8 & - & - \\
& IPA & 28.4 & $2.32_{\mathrm{ads}} / 2.30_{\mathrm{des}}$ & $1.36_{\mathrm{abs}} / 1.38_{\mathrm{des}}$ \\
& Methanol & 27.6 & - & - \\
${ }^{t} \mathrm{BuMe}_{2} \mathrm{SiCl}$ & Toluene & 37.3 & $2.76_{\mathrm{ads}} / 2.66_{\mathrm{des}}$ & $1.50_{\mathrm{ads}} / 1.54_{\mathrm{des}}$ \\
& Water & 35.8 & - & - \\
& IPA & 38.7 & $2.66_{\mathrm{ads}} / 2.62_{\mathrm{des}}$ & $1.02_{\mathrm{ads}} / 1.12_{\mathrm{des}}$ \\
& Methanol & 36.8 & - & -
\end{tabular}

there is very little evidence that it has modified the pores of the film. In particular, the pore volume fraction measured with this film (37\%) is to within error the same as for an untreated film $(38 \%)$ whereas the other two treated films have pore volume fractions nearer $30 \%$. In addition this film, like untreated films, shows no difference in the porosity measured with water and the other three solvents. Whilst ${ }^{t} \mathrm{BuMe}_{2} \mathrm{SiCl}$ was able to penetrate the pores of the powered mesoporous silica to some degree, it is clear that the pores in the films are significantly smaller $(\sim 1.6 \mathrm{~nm})$ compared to the powered samples $(\sim 3 \mathrm{~nm})$.

The presence of residual CTAB in the pores after grafting, as shown by the NMR and combustion analysis, could potentially contribute to the hydrophobic character of the pores. However the porosimetry measurements show that this is not the case. The porosity measured with water in the ungrafted film, which contains the most CTAB, is $40.1 \%$, while the equivalent porosity measurement in the $\mathrm{Me}_{3} \mathrm{SiCl}$ grafted film, the film which contains almost no CTAB, shows $7.7 \%$. This is also shown for the other grafted films having greater hydrophobicity than the ungrafted film. This indicates that the presence of CTAB has no influence on the hydrophobic character of the films.

All of the evidence taken together suggests that for pores in the films the diameter that the size of the grating agent is critical to the successful grating of the pores. This constitutes a novel route to control pore sizes in vertically aligned hexagonal mesoporous silica films, opening new possibilities for fine tuning of the molecular sieving properties of such oriented membranes, ${ }^{65}$ and to control pore wall chemistry with the possibility of allowing ingress of reagents or solvents for electrodeposition.

\section{Conclusions}

Hexagonal mesoporous silica powder and vertically aligned films have been synthesized using TEOS and CTAB surfactant.
Both exhibited good hexagonal order in the pore structures and similar pore spacings. The nitrogen sorption measurements showed a surface area of approximately $1100 \mathrm{~m}^{2} \mathrm{~g}^{-1}$ for the powder. The ellipsometric porosimetry measurements gave a pore diameter of approximately $1.4 \mathrm{~nm}$ using the Dubinin-Rasushkevich model and $2.7 \mathrm{~nm}$ using a modified Kelvin equation. The former was consistent with previous TEM evidence.

The mesoporous silica powders and films were successfully grafted with various silane reagents. The SAXS measurements showed little difference in the pore structure between the ungrafted and grafted mesoporous films and powders. The ${ }^{29} \mathrm{Si}$ NMR analysis showed that the grafted powders contained extra Si environments corresponding to the silane groups grafted onto the pore walls. The CHN analysis indicated that some CTAB surfactant was still present in the pores of the ungrafted silica powders, but was displaced by the grafting with silane groups. DFT calculations showed a decrease in the pore diameter between the ungrafted and the grafted powders. A general trend was observed for the different silane grafting reagents that the reagents with the smallest steric bulk gave the greater coverage of the pore walls and largest grafted species peaks in the ${ }^{29} \mathrm{Si}$ NMR spectra. This indicates that the bulkier reagents are unable to fully penetrate the pore and fully graft the inside of the pore wall.

Ellipsometric porosimetry of the grafted mesoporous silica films showed that the penetration of water greatly decreased when grafted with the smaller silane reagents such as $\mathrm{Me}_{3} \mathrm{SiCl}$, which was clearly effective in making the pore walls hydrophobic. However there was no change when grafting with the larger silane reagents such as ${ }^{t} \mathrm{BuMe}_{2} \mathrm{SiCl}$, and evidence for partial grafting at the top of the pores with $\mathrm{PhMe}_{2} \mathrm{SiCl}$, further indicating that the size of the grafting agent affects the degree of grafting undertaken in the pore structure. Surfactant persisted in the pores after Soxhlet extraction, but this did not significantly affect hydrophobicity. The smaller pores in the silica films relative to the powders resulted in a higher degree of exclusion of the larger grafting agents.

\section{Acknowledgements}

The authors thank EPSRC for funding under the Supercritical Fluids Electrodeposition project (SCFED, EP/I033394/1) and for funding the Smartlab diffractometer (EP/K00509X/1 and EP/ K009877/1). The SCFED Project (www.scfed.net) is a multidisciplinary collaboration of British universities investigating the fundamental and applied aspects of supercritical fluids. Marina Carravetta thanks the Royal Society for a University Research Fellowship.

\section{References}

1 I. K. Mbaraka and B. H. Shanks, J. Catal., 2005, 229, 365-373. 2 A. Walcarius, Chem. Soc. Rev., 2013, 42, 4098-4140.

3 B. J. Melde, B. J. Johnson and P. T. Charles, Sensors, 2008, 8, 5202-5228. 
4 R. I. Nooney, D. Thirunavukkarasu, Y. Chen, R. Josephs and A. E. Ostafin, Chem. Mater., 2002, 14, 4721-4728.

5 T. Maschmeyer, F. Rey, G. Sankar and J. M. Thomas, Nature, 1995, 378, 159-162.

6 J. Zhang, Z. Ma, J. Jiao, H. Yin, W. Yan, E. W. Hagaman, J. Yu and S. Dai, Microporous Mesoporous Mater., 2010, 129, 200209.

7 F. Hoffmann, M. Cornelius, J. Morell and M. Fröba, Angew. Chem., Int. Ed., 2006, 45, 3216-3251.

8 D. J. Macquarrie, Green Chem., 1999, 195-198.

9 J. S. Beck, J. C. Vartuli, W. J. Roth, M. E. Leonowicz, C. T. Kresge, K. D. Schmitt, C. T. W. Chu, D. H. Olson, E. W. Sheppard, S. B. McCullen, J. B. Higgins and J. L. Schlenker, J. Am. Chem. Soc., 1992, 114, 10834-10843.

10 R. Luque, A. M. Balu, J. M. Campelo, M. D. Gracia, E. Losada, A. A. R. Pineda and J. C. Serrano-Ruiz, in Catalysis: Volume 24, ed. J. J. Spivey and M. Gupta, RSC Publishing, Cambridge, UK, 2012, pp. 253-280.

11 N. Lashgari, A. Badiei and G. M. Ziarani, Nanochemistry Research, 2016, 1, 127-141.

12 Y. G. Jin, S. Z. Qiao, Z. P. Xu, Z. Yan, Y. Huang, J. C. Diniz da Costa and G. Q. Lu, J. Mater. Chem., 2009, 19, 2363.

13 A. Walcarius, Electroanalysis, 2015, 27, 1303-1340.

14 M. Etienne, L. Zhang, N. Vila and A. Walcarius, Electroanalysis, 2015, 27, 2028-2054.

15 L. Bois, A. Bonhommé, A. Ribes, B. Pais, G. Raffin and F. Tessier, Colloids Surf., A, 2003, 221, 221-230.

16 A. Walcarius and L. Mercier, J. Mater. Chem., 2010, 20, 4478.

17 A. Nomura and C. W. Jones, ACS Appl. Mater. Interfaces, 2013, 5, 5569-5577.

18 I. Andreou, H. Amenitsch, V. Likodimos, P. Falaras, P. Koutsoukos and E. Leontidis, Materials, 2013, 6, 14671484.

19 Y.-F. Lee, K.-H. Chang, C.-Y. Chu, H.-L. Chen and C.-C. Hu, RSC Adv., 2011, 1, 401-407.

20 D. Grosso, F. Cagnol, G. J. de A. A. Soler-Illia, E. L. Crepaldi, H. Amenitsch, A. Brunet-Bruneau, A. Bourgeois and C. Sanchez, Adv. Funct. Mater., 2004, 14, 309-322.

21 P. Innocenzi and L. Malfatti, Chem. Soc. Rev., 2013, 42, 41984216.

22 A. Walcarius, E. Sibottier, M. Etienne and J. Ghanbaja, Nat. Mater., 2007, 6, 602-608.

23 A. Walcarius and A. Kuhn, TrAC, Trends Anal. Chem., 2008, 27, 593-603.

24 M. Etienne, A. Goux, E. Sibottier and A. Walcarius, J. Nanosci. Nanotechnol., 2009, 9, 2398-2406.

25 Y. Guillemin, J. Ghanbaja, E. Aubert, M. Etienne and A. Walcarius, Chem. Mater., 2014, 26, 1848-1858.

26 G. Herzog, E. Sibottier, M. Etienne and A. Walcarius, Faraday Discuss., 2013, 164, 259-273.

27 C. Robertson, R. Beanland, S. A. Boden, A. L. Hector, R. J. Kashtiban, J. Sloan, D. C. Smith and A. Walcarius, Phys. Chem. Chem. Phys., 2015, 17, 4763-4770.

28 Z. Teng, G. Zheng, Y. Dou, W. Li, C.-Y. Mou, X. Zhang, A. M. Asiri and D. Zhao, Angew. Chem., Int. Ed. Engl., 2012, 51, 2173-2177.
29 M. Hara, S. Nagano and T. Seki, J. Am. Chem. Soc., 2010, 132, 13654-13656.

30 Y. Guillemin, M. Etienne, E. Aubert and A. Walcarius, J. Mater. Chem., 2010, 20, 6799-6807.

31 A. Goux, M. Etienne, E. Aubert, C. Lecomte, J. Ghanbaja and A. Walcarius, Chem. Mater., 2009, 21, 731-741.

32 E. M. Björk, Mesoporous Building Blocks - Synthesis and Characterization of Mesoporous Silica Particles and Films, PhD thesis, Linkoping University, 2013.

33 D. J. Macquarrie, Top. Catal., 2009, 52, 1640-1650.

34 P. N. Bartlett, J. Burt, D. A. Cook, C. Y. Cummings, M. W. George, A. L. Hector, M. M. Hasan, J. Ke, W. Levason, D. Pugh, G. Reid, P. W. Richardson, D. C. Smith, J. Spencer, N. Suleiman and W. Zhang, Chem.-Eur. J., 2015, 302-309.

35 P. N. Bartlett, C. Y. Cummings, W. Levason, D. Pugh and G. Reid, Chem.-Eur. J., 2014, 20, 5019-5027.

36 C. Y. Cummings, P. N. Bartlett, D. Pugh, G. Reid, W. Levason, M. M. Hasan, A. L. Hector, J. Spencer and D. C. Smith, J. Electrochem. Soc., 2015, 162, D619-D624.

37 N. Vilà, J. Ghanbaja, E. Aubert and A. Walcarius, Angew. Chem., Int. Ed. Engl., 2014, 53, 2945-2950.

38 N. Vilà, J. Ghanbaja and A. Walcarius, Adv. Mater. Interfaces, 2016, 3, 1500440.

39 M. Rafiee, B. Karimi, S. Farrokhzadeh and H. Vali, Electrochim. Acta, 2013, 94, 198-205.

40 S. Brunauer, P. H. Emmett and E. Teller, J. Am. Chem. Soc., 1938, 60, 309-319.

41 J. Landers, G. Y. Gor and A. V. Neimark, Colloids Surf., A, 2013, 437, 3-32.

42 A. M. Puziy, O. I. Poddubnaya, B. Gawdzik and M. Sobiesiak, Adsorption, 2016, 22, 459-464.

43 ISO 15901-32007, Pore size Distrib. porosity solid Mater. by Mercur. Porosim. gas Adsorpt. 3 Anal. micropores by gas Adsorpt, https//www.iso.org/obp/ui/\#isostdiso15901-3ed1v1en,.

44 N. A. Seaton, J. P. R. B. Walton and N. Quirke, Carbon, 1989, 27, 853-861.

45 M. R. Baklanov, K. P. Mogilnikov, V. G. Polovinkin and F. N. Dultsev, J. Vac. Sci. Technol., B: Microelectron. Nanometer Struct.-Process., Meas., Phenom., 2000, 18, 1385.

46 C. Lastoskie, K. E. Gubbins and N. Quirke, J. Phys. Chem., 1993, 97, 4786-4796.

47 M. Luisa Ojeda, J. Marcos Esparza, A. Campero, S. Cordero, I. Kornhauser and F. Rojas, Phys. Chem. Chem. Phys., 2003, 5, 1859.

48 A. V. Neimark and P. I. Ravikovitch, Microporous Mesoporous Mater., 2001, 44, 697-707.

49 M. Thommes, B. Smarsly, M. Groenewolt, P. I. Ravikovitch and A. V. Neimark, Langmuir, 2006, 22, 756-764.

50 P. I. Ravikovitch, a. Vishnyakov and a. V. Neimark, Phys. Rev. E: Stat., Nonlinear, Soft Matter Phys., 2001, 64, 011602.

51 A. Bourgeois, Y. Turcant, C. Walsh and C. Defranoux, Adsorption, 2008, 14, 457-465.

52 M. Etienne, Y. Guillemin, D. Grosso and A. Walcarius, Anal. Bioanal. Chem., 2013, 405, 1497-1512.

53 A. Wang and T. Kabe, Chem. Commun., 1999, 2067-2068. 
54 M. Gru, K. K. Unger, A. Matsumoto and K. Tsutsumi, Microporous Mesoporous Mater., 1999, 27, 207-216.

55 T. Asefa and Z. Tao, Can. J. Chem., 2012, 90, 1015-1031.

56 C. Boissiere, D. Grosso, S. Lepoutre, L. Nicole, A. B. Bruneau and C. Sanchez, Langmuir, 2005, 21, 12362-12371.

57 M. Thommes, Chem.-Ing.-Tech., 2010, 82, 1059-1073.

58 R. Simonutti, A. Comotti, S. Bracco and P. Sozzani, Chem. Mater., 2001, 13, 771-777.

59 P. Sutra, F. Fajula, D. Brunel, P. Lentz, G. Daelen and J. B. Nagy, Colloids Surf., A, 1999, 158, 21-27.
60 T. A. Blinka, B. J. Helmer and R. West, in Advances in Organometallic Chemistry, ed. F. G. A. Stone and R. West, Academic Press Inc., New York, 1984, pp. 193-218.

61 C. Henrist, C. Vogels, A. Rulmont and R. Cloots, New J. Chem., 2005, 29, 1017-1021.

62 M. H. Lim and A. Stein, Chem. Mater., 1999, 11, 3285-3295.

63 L. Mercier and T. J. Pinnavaia, Chem. Mater., 2000, 12, 188196.

64 R. J. P. Corriu, E. Lancelle-Beltran, A. Mehdi, C. Reyé, S. Brandès and R. Guilard, Chem. Mater., 2003, 15, 3152-3160.

65 N. Vilà, E. André, R. Ciganda, J. Ruiz, D. Astruc and A. Walcarius, Chem. Mater., 2016, 28, 2511-2514. 